\title{
LA LEXICOGRAFÍA DE INTERNET Y EL DISEÑO DE DICCIONARIOS PEDAGÓGICOS ESPECIALIZADOS: EL DICCIONARIO DE COMERCIO INTERNACIONAL ESPAÑOL-INGLÉS
}

\author{
Begoña Inmaculada García Llamas \\ Universidad Nacional de Educación a Distancia
}

\begin{abstract}
RESUMEN: El presente trabajo forma parte de un proyecto de investigación más general que abarca la propuesta para la elaboración de un diccionario de Comercio Internacional dentro del marco teórico de la Teoría Funcional de la Lexicografía. Este proyecto, que se expondrá en forma de tesis doctoral, propone la creación de un diccionario electrónico pedagógico especializado en lenguas inglesa y española para alumnos universitarios españoles. Presentamos a continuación un resumen de aquellas secciones del estudio que tienen en común el enfoque pedagógico de la propuesta: la selección de categorías de información y dos elementos estructurales (Introducción Sistemática y Guía de Uso) relevantes para el usuario.

Tras una introducción en la que presentamos el estado de la cuestión, resumimos el marco teórico en el que se circunscribe nuestra propuesta. A continuación exponemos los hechos que la justifican desde un punto de vista pedagógico junto con nuestros objetivos y un listado de categorías de información y elementos estructurales relevantes para nuestro usuario y que consideramos decisivos en el proceso de formación del alumno.

Por último, se ofrecen ilustraciones de una posible interfaz del diccionario en distintas fases de una consulta.
\end{abstract}

PALABRAS CLAVE: Teoría Funcional de la Lexicografía, diccionario pedagógico, usuario/alumno, necesidades lexicográficamente relevantes, situación social o extralexicográfica, datos lexicográficos relevantes.

\section{E-LEXICOGRAPHY AND THE DESIGN OF PEDAGOGICALLY- ORIENTED SPECIALIZED DICTIONARIES: THE DICCIONARIO DE COMERCIO INTERNACIONAL ESPAÑOL-INGLÉS}

ABSTRACT: The present article represents a part of a more comprehensive project which entails the proposal for the elaboration of an International Trade 
Dictionary inside the theoretical framework of the Functional Theory of Lexicography. This project, which will be presented as a Ph dissertation, proposes the creation of a specialized electronic pedagogical dictionary in English and Spanish for Spanish university students. We present here the summary of all those sections in the study which focus on the pedagogic approach of the proposed reference tool. Specifically: the selection of information categories and the elaboration of two structural elements (Systematic Introduction and User's Guide) considered to be highly relevant for the user.

After a brief overview of the state-of-the-arts, we summarize the theoretical framework where our proposal of a dictionary is included. Next, we state the pedagogical facts that justify our work and our objectives together with a list of the information categories and structural elements considered as relevant for our target user.

Finally, we present a set of illustrations showing the possible layout for the dictionary interface in different phases of the search process.

KEYWORDS: Function Theory of Lexicography, learner's dictionary, user/student, lexicographically relevant needs, social or extralexicographic situation, lexicographically relevant data.

\section{Introducción}

El alumno universitario español en general y el de Comercio Internacional en particular, desarrolla su proceso formativo en múltiples situaciones, siendo tres de ellas las más relevantes: las clases presenciales en grupo, el autoestudio, y las sesiones tutoriales individualizadas. A esta diversidad de situaciones sociales inherentes a los estudios universitarios se suman tres hechos especialmente relevantes para la elaboración de un diccionario pedagógico como el que aquí describimos: por un lado, el proceso de innovación tecnológica especialmente intenso en el ámbito universitario; por otro, el imparable proceso de globalización y la importancia concedida por las instituciones ${ }^{1}$ a la adaptación del alumnado a este proceso (materializado en la implantación de los Acuerdos de Bolonia) y por último, la misma naturaleza del campo de especialización del Comercio Internacional que precisa que todo aquel que quiera llegar al grado de experto domine tanto las nuevas tecnologías de la información como la lengua inglesa, en la que se llevan a cabo la mayor parte de sus actividades.

Nuestra propuesta se circunscribe dentro de este ámbito pedagógico, buscando ofrecer al alumno (y al profesor) de Comercio Internacional una herramienta de consulta que facilite la adquisición de conocimientos y vocabulario especializados en una

1. Ver http://www.senado.es/legis8/expedientes/661/impresion /661000328.html, $\mathrm{n}^{\mathrm{o}}$ de expediente 661/000328). 
segunda lengua dentro de un sistema de estudios cuya estructura y contenidos han cambiado radicalmente en los últimos años. En concreto, el Comercio Internacional, anteriormente incluido como parte de algunas licenciaturas (aparte de su tratamiento más específico en las Escuelas de Comercio), es hoy una materia de estudio de tales proporciones e importancia que ha pasado a constituir un grado independiente en algunas universidades ${ }^{2}$. A la vista de esta situación, este trabajo tiene tres objetivos: a) definir con precisión las necesidades lexicográficamente relevantes de tipo cognitivo y comunicativo que surgen en el proceso formativo de un alumno en situación de recepción de textos escritos; b) proponer recursos para la formación del alumno en el manejo de nuevas herramientas lexicográficas y c) proponer la creación de una herramienta que facilite a los profesores su labor docente.

La posibilidad actual de diseñar herramientas de referencia en formato digital y con acceso a Internet, ha abierto el camino a la creación de obras que se adaptan más adecuadamente a las necesidades reales del estudiante en las situaciones sociales mencionadas anteriormente. Sin embargo, lo que no aporta la tecnología son las directrices para crear el contenido de esas obras. Adhiriéndonos a la afirmación de Henning Bergengholtz ${ }^{3}$ de que no hay nada más práctico que una buena teoría, hemos seguido las directrices que nos proporciona la Teoría Funcional de la Lexicografía y su versión más actualizada, la Moderna Teoría Funcional de la Lexicografía, para la elaboración de nuestra propuesta de diccionario pedagógico.

En el presente artículo, tras exponer brevemente los principios generales del marco teórico en el que se circunscribe nuestra propuesta de diccionario, definimos los parámetros funcionales que conforman la base de nuestro proyecto y en un tercer apartado ofrecemos un ejemplo de lo que sería la interfaz del diccionario en varios momentos de una búsqueda. Cerramos la exposición con las conclusiones alcanzadas tras la elaboración de nuestro trabajo de investigación.

\section{Marco teórico: principios generales de la Teoría Funcional de la Lexicografía}

El estudio que aquí presentamos se circunscribe dentro de la Teoría Funcional de la Lexicografía definida por Bergenholtz y Tarp $(2003,2004,2005)$ y Tarp (2008) en el Aarhus School of Business's Centre for Lexicography. Esta teoría surge

2. Por ejemplo: Universidad Complutense http://www.ucm.es/?a=estudios\&d=muestragrado\& idgr $=14$.

3. En Nielsen y Tarp (2009). 
como alternativa y superación de paradigmas anteriores tales como El esbozo de una teoría general de la Lexicografía formulada por el lexicógrafo ruso Lev Scherba en 1940 y la Teoría general de la Lexicografía desarrollada por el lexicógrafo alemán Herbert Ernst Wiegand . Desde sus inicios, la Teoría Funcional se ha ido modificando con el fin de dar cabida a nuevos planteamientos teóricos, normalmente basados en la práctica lexicográfica. Uno de los cambios más llamativos hace referencia a los diccionarios de Internet pensados para individualizar las búsquedas y la recreación y re-representación de los datos que centrarán el debate en los próximos años (Fuertes-Olivera y Tarp, 2014).

La Teoría Funcional de la Lexicografía parte de la definición de cuatro conceptos básicos: cuál es la verdadera esencia de la Lexicografía, quién es el destinatario/usuario de la obra lexicográfica, cuáles son las necesidades lexicográficamente relevantes del usuario y en qué situaciones extralexicográficas surgen las necesidades concretas de un usuario concreto. Todo esto queda resumido en la definición de la Lexicografía expuesta por Tarp, según la cual, la Lexicografía es:

[...] la teoría y práctica de las herramientas de uso que se caracterizan por su capacidad para facilitar un rápido y fácil acceso al tipo específico de datos de los cuales un tipo específico de usuarios pueden extraer el tipo específico de información que les permita satisfacer el tipo específico de necesidades (lexicográficamente relevantes) que tengan en un tipo específico de situación social extra-lexicográfica (Tarp 2008: 4).

Esta definición tiene múltiples implicaciones: en primer lugar, supone la desvinculación de la Lexicografía de la Lingüística Aplicada. La Lexicografía pasa de ser una disciplina auxiliar a ser una ciencia multidisciplinar en la que la elaboración de las herramientas lexicográficas (sean o no diccionarios) es llevada a cabo por lexicógrafos y otros profesionales de diversos campos. La siguiente implicación es el cambio en el punto de partida al elaborar las obras de referencia. En la Lexicografía tradicional se parte de la tipología de la obra mientras que en la Teoría Funcional el punto de partida es la definición de la situación social extralexicográfica en la que se encuentra el usuario potencial. La Teoría Funcional distingue cuatro tipos fundamentales de situaciones relevantes para la Lexicografía, a saber: las comunicativas, las cognitivas, las interpretativas y las operacionales ${ }^{4}$. Una vez establecida la situación social en la que

4. Tarp (2010); Fuertes-Olivera y Bergenholtz (2011); Tarp (2010:39-53): "Functions of Specialised Learners' Dictionaries" en Pedro A. Fuertes-Olivera (ed.). Specialised Dictionaries for Learners. Berlín y Nueva York: De Gruyter, pp. 39-53. 
surgirán las necesidades del usuario, la Teoría Funcional estudia las correspondientes características relevantes de los posibles usuarios.

La siguiente implicación es de carácter práctico, y se refiere al cambio en los criterios de selección de las entradas de las obras de referencia. La Teoría Funcional aboga por el empleo del criterio de relevancia que se traduce en una selección de lemas vinculada a las necesidades del usuario concreto en una situación concreta, con lo que la calidad de la obra se medirá no por el número de entradas sino por el número de entradas realmente útiles para el usuario. Este enfoque orientado al usuario conlleva igualmente la desaparición de la distinción entre diccionarios lingüísticos y enciclopedias ya que la Teoría de las Funciones asume que rara vez el usuario va a necesitar un único tipo de datos para satisfacer sus necesidades de información por lo que la tipología tradicional debe sustituirse por una nueva basada en las funciones lexicográficas. ${ }^{5}$ Por último, la definición de la Lexicografía aquí defendida se refiere a la capacidad de las obras de referencia de proporcionar un acceso rápido y fácil a los datos por parte del usuario.

\section{Definición de los parámetros funcionales}

En el presente apartado exponemos los resultados del proceso de investigación conducente a la definición de las necesidades lexicográficas reales del usuario en cuestión. Dicho proceso comprende varias fases. En primer lugar se definieron tanto la situación extralexicográfica en la que surgían las necesidades lexicográficamente relevantes del usuario así como el perfil de dicho usuario. A continuación se localizaron los principales diccionarios impresos de Comercio Internacional adecuados a las necesidades de los alumnos descritos en el estudio. El tercer paso consistió en la búsqueda en dichos diccionarios de una serie de términos propios del campo del Comercio Internacional y se constató la insuficiencia de dichas obras impresas para satisfacer las necesidades lexicográficamente relevantes del usuario. El siguiente paso fue la localización en Internet de aquellas obras lexicográficas y terminológicas con funcionalidad similar a la de los diccionarios impresos con el fin de averiguar si la información que no se había hallado en las obras impresas aparecía en las obras electrónicas.

Fuertes-Olivera y Bergenholtz (eds.) (2011): E-Lexicography. The Internet, Digital Initiatives and Lexicography. Londres y Nueva York: Continuum.

5. Tarp (2010): "Functions of Specialised Learners' Dictionaries" en Pedro A. Fuertes-Olivera (ed.). Specialised Dictionaries for Learners. Berlín y Nueva York: De Gruyter, p. 40. 
El último paso consistió en evaluar los resultados de las búsquedas de información, de manera que se pudiese concluir de forma objetiva qué tipo de obra era más adecuada para satisfacer las necesidades del usuario y si existía una justificación real o no para la elaboración de una obra como la que se propone.

Las conclusiones principales de este proceso de investigación fueron, por un lado, que ninguna de las obras analizadas satisfacía todas las necesidades del usuario y, por otro lado, que el medio más adecuado para elaborar una obra que satisficiera adecuadamente dichas necesidades era el electrónico.

\subsection{Situación extralexicográfica}

El usuario objeto de este estudio deberá hacer frente a múltiples necesidades asociadas a varias situaciones extralexicográficas relevantes para la Lexicografía. El presente estudio se centra en las necesidades cognitivas así como en las comunicativas, incidiendo de forma más detallada en aquellas surgidas en situación de recepción de textos orales y escritos. En un futuro tenemos previsto ampliar las funciones del diccionario, en línea con las propuestas actuales que diferencian las bases de datos lexicográficas de los diccionarios. Dicha ampliación aportaría soluciones a las necesidades lexicográficas surgidas en situación de producción de textos orales y escritos.

\subsection{Descripción del usuario}

El usuario objeto de este estudio se corresponde con un alumno de una universidad española ${ }^{6}$ proveniente del Bachillerato y que cursa estudios relacionados con el Comercio Internacional (o Comercio Exterior). Actualmente esto incluye a los estudiantes del Grado en Comercio impartido en varias universidades españolas así como a los de diversas titulaciones oficiales en las que se incluye el estudio de esta área del conocimiento, como pueden ser Economía, Administración y Dirección de Empresas y Ciencias Empresariales entre otras.

Para ayudar a identificar las necesidades lexicográficas del alumno como usuario potencial de un diccionario, Tarp (2008:18) propone una serie de preguntas que nos dan un perfil más detallado de lo que será el destinatario de nuestra propuesta. Allí

6. "Real Decreto 1393/2007, de 29 de octubre, por el que se establece la ordenación de las enseñanzas universitarias oficiales”. B.O.E. núm. 260. 
también afirma que esta es una lista abierta en la que el lexicógrafo puede ir añadiendo más preguntas según sea necesario. En concreto nosotros proponemos tres preguntas adicionales que creemos relevantes para la elaboración del diccionario:

- ¿Cuál es la formación lexicográfica del usuario en su lengua materna?

- ¿Cuál es la formación lexicográfica del usuario en la segunda lengua?

- ¿Cuál es su formación y experiencia en el campo de las nuevas tecnologías de la información y su posibilidad de acceso a ellas?

A partir de estas preguntas, definimos a nuestro usuario como:

a) un alumno con español como lengua nativa;

b) con un grado de dominio de la lengua española detallado en el currículum oficial ${ }^{7}$ para el Bachillerato marcado por el Ministerio de Educación, Política Social y Deporte;

c) usuario de nivel B1 de conocimientos en lengua inglesa según el Marco Común Europeo de Referencia para las Lenguas;

d) con cierta especialización en la rama de Humanidades y Ciencias Sociales, adquiridos a lo largo de la formación secundaria obligatoria y el Bachillerato;

e) su motivación para aprender el idioma es la necesidad de superar las asignaturas que se imparten en la titulación, así como la necesidad de demostrar un nivel de inglés adecuado para completar o complementar sus estudios en el extranjero y/o el deseo de aumentar sus posibilidades de incorporarse al mundo laboral una vez terminados sus estudios;

f) está inmerso en un proceso sistemático (no espontáneo) de aprendizaje de la lengua inglesa;

g) el aprendizaje de la segunda lengua lo lleva a cabo o como asignatura de Lengua Inglesa ligada a los estudios propios del grado o la titulación en cuestión o como lengua usada en las sesiones teóricas de diversas asignaturas impartidas en el aula por un profesor, así como en los diversos materiales didácticos y bibliográficos que maneja durante sus estudios;

7. Para ver en detalle el currículum marcado por el Ministerio de Educación, ver el "Real Decreto 1467/2007, de 2 de noviembre, por el que se establece la estructura del y se fijan sus enseñanzas mínimas." 
h) tiene contacto con su lengua materna durante el proceso de aprendizaje,

i) usa, tanto libros de texto para el aprendizaje de la lengua inglesa como diversos materiales de apoyo en lengua inglesa;

j) sigue la metodología elegida libremente por cada profesor, si bien esta se debe adecuar a los criterios de los Acuerdos de Bolonia;

k) el aprendizaje está ligado a la materia de Comercio Internacional;

1) tiene práctica en el uso de diccionarios tanto impresos como electrónicos, pero desconoce mucha de la nomenclatura lexicográfica que encuentra en ellos, con lo que toda la información que podría obtener a partir de esos datos no llega a materializarse ${ }^{8}$;

m) hace uso de Internet pero no necesariamente tiene conexión en su domicilio, aunque sí puede conectarse en las bibliotecas universitarias donde sí hay conexión para los alumnos y en algunas se ofrece préstamo de ordenadores portátiles.

n) posee un nivel suficiente para manejar una herramienta electrónica como la que proponemos en esta tesis; pero, desde un punto de vista pedagógico, no damos por supuesto el hecho de que todos los alumnos son igualmente diestros ni están igualmente motivados para el manejo de estas tecnologías cuando el objetivo no es el ocio sino el estudio.

\subsection{Necesidades lexicográficas}

Una vez analizada y definida la situación concreta en que se encuentra el usuario/alumno tal y como lo hemos definido, concretamos las necesidades lexicográficas del mismo.

3.3.1. Elección del término de búsqueda: en primer lugar, el alumno necesita tener un acceso fácil a los datos y el primer paso para ello es la elección del término de búsqueda.

- Términos y términos multipalabra: en nuestra propuesta seguimos el ejemplo de una obra clave realizada en el seno de la Teoría Funcional de la Lexicogra-

8. Por ejemplo: Martínez, A. (2001). 
fía, El Diccionario de Contabilidad Inglés-Español ${ }^{9}$, que proporciona una herramienta de búsqueda idónea para solucionar el problema de acceso a datos de este tipo. Por un lado permite buscar un término que conste de una sola palabra eligiendo la opción is (es) y por otro, incluye las opciones contains (contiene), begins with (comienza por) y ends with (termina por) para buscar términos multipalabra ordenados alfabéticamente. De esta manera solucionamos el problema de elegir por dónde buscar términos como ad valorem tariff, differentiated tariff items, o tariff premium rate.

- Homónimos y homógrafos: como segundo paso para la búsqueda el usuario debe tener claro a qué realidad conceptual corresponde el término que desconoce. Proponemos aquí seguir el ejemplo de la obra de referencia Wikipedia que ofrece una herramienta llamada página de desambiguación en la que ofrece un listado con todos los homónimos y homógrafos del término acompañados de una breve indicación que establece la diferencia esencial entre unos y otros.

- Términos polisémicos: una vez que el usuario ha identificado el homónimo u homógrafo al que se refiere su consulta, puede tener que decidir qué acepción del mismo es la que busca si se trata de un término polisémico. Para ello, el diccionario usaría el mismo método que para la diferenciación de homónimos y homógrafos. Cada acepción aparecerá brevemente explicada en inglés con una oración completa que pueda ser traducida al español mediante la herramienta en español.

3.3.2. Categorías de información. En este apartado exponemos las categorías de información que consideramos relevantes para nuestro usuario y las presentamos de la forma en que irían apareciendo en el diccionario electrónico que proponemos. Este orden responde a criterios pedagógicos y se basa en la dosificación de la cantidad de datos proporcionados en cada pantalla (o paso de la búsqueda) y en el uso de las lenguas inglesa y española con el fin de aumentar la competencia del alumno en inglés pero con la opción (siempre disponible pero no siempre más cómoda) de acceder a los datos en español.

- Equivalente cognitivo en español: en el caso de términos polisémicos se proporcionará un equivalente para cada acepción del término, las cuales vendrán separadas, numeradas y precedidas de su marca de materia. En nuestro estudio solo nos centramos en la situación de recepción, pero debe apuntarse que para una si-

9. Disponible en http://www.lemma.com. 
tuación de producción, cada equivalente debe contar con sus correspondientes aclaraciones en cuanto a restricciones pragmáticas si las hubiera y sus características gramaticales. Lo que sí consideramos imprescindible en la situación de recepción es la inclusión de aclaraciones referentes a diferencias culturales entre los distintos ámbitos geográficos de aplicación del término. Bergengholtz y Nielsen (2006:94-134) explican este hecho introduciendo el término de "materia culturalmente dependiente".

Debido al carácter pedagógico de nuestro diccionario, evitamos ofrecer el equivalente en español en la primera pantalla ${ }^{10}$. Si bien es cierto que esto puede ahorrar tiempo al alumno, también es cierto que estaríamos perdiendo la oportunidad de que el alumno profundice de forma sencilla pero eficaz en el área de especialización, así como que vaya reforzando y ampliando sus conocimientos de lenguaje especializado en inglés.

- Explicación ${ }^{11}$ : se proporcionará una explicación adaptada al nivel de conocimientos en el área de Comercio y en la segunda lengua. Como señalan FuertesOlivera y Arribas Baño (2008:141), el hecho de que se proporcione gran cantidad de los datos en el diccionario en la segunda lengua (inglés en este caso) ayuda al usuario a aprender dicha lengua. Coincidimos con Nielsen y Almind (2011:156157) en incluir las definiciones como oraciones completas usando lenguaje natural para que los usuarios puedan transformar fácilmente los datos en información mediante un proceso mental. Esta explicación podrá ser traducida al L1 mediante un vínculo del tipo en español e incluirá el equivalente cognitivo proporcionado anteriormente. Por otro lado, el alumno de cursos avanzados podrá acceder a una explicación más detallada y técnica por medio de un hipervínculo que le remitirá a la correspondiente sección de la Introducción Sistemática. Esto da respuesta a la afirmación de Botha (2011:76) según la cual “...the user would prefer not to have to read through much detail if only a single fact is required; neither would the user like to have to consult multiple sources to get to the detail required for a more comprehensive view on a topic".

- Marca de materia: para elaborar el listado de subáreas que debe abarcar esta obra, partimos del perfil del usuario y aplicando el criterio de relevancia lle-

10. Compartimos la decisión de Fernández y Faber en relación al diccionario Online Hypertextual Dictionary of Architecture and Construction donde el equivalente en español solo está disponible si se pincha sobre el botón correspondiente.

11. Utilizamos el término "explicación” como sinónimo de "definición”. 
gamos como resultado a la siguiente lista de subáreas: Administración, Arbitraje, Auditoría, Comunicación y Negociación, Contabilidad, Contratación, Derecho, Documentación, Economía, E-comercio, Gestión Empresarial, Estadística, Exportaciones, Fiscalidad y Tributación, Gestión Financiera, Importaciones, Informática, Instituciones, Logística, Gestión de Calidad del Medio Ambiente, Macroeconomía, Marketing, Matemáticas, Investigación de Mercados, Microeconomía, Prevención de Riesgos Laborales, Política, Publicidad y Relaciones Públicas, Recursos Humanos, Relaciones Internacionales, Seguros, Sociología, Ventas.

La inclusión de este dato junto a cada acepción de un término tiene una doble función: por un lado, el alumno sabrá a qué parte de la disciplina que está estudiando afecta este concepto, lo cual, desde un punto de vista pedagógico es recomendable ya que en ocasiones el alumno adquiere conocimientos puntuales pero pierde la visión de conjunto imprescindible para dominar la materia. Por otro lado, cada marca de materia se corresponde con una sección de la Introducción Sistemática, con lo que se convierte en una herramienta de acceso a más datos al estar configurada como un hipervínculo que llevará al alumno a la sección correspondiente.

- Ilustraciones: se propone la inclusión de ilustraciones siempre que sea posible por su doble valor pedagógico: explicativo y motivador.

- Información enciclopédica: el usuario podrá acceder a este tipo de información a través de dos partes del diccionario:

a) en la Introducción Sistemática ${ }^{12}$ : esta incluye parte de la información presente en las entradas del diccionario así como información adicional. Está organizada en secciones que se corresponden con las marcas de materia adjuntas a las entradas y que cubren todas las subáreas del Comercio Internacional.

b) en páginas de Internet seleccionadas: pinchando una opción del tipo explanation in full adjunta a cada término.

En un futuro cercano están previstas 27 opciones de búsquedas en línea con las propuestas actuales que diferencian las bases de datos lexicográficas de los diccionarios. En este marco, el lexicógrafo está obligado a incluir también ejemplos, sinónimos, pronunciación, términos relacionados, colocaciones y otras catego-

12. Ver apartado 2.3.3. para una explicación más detallada de este punto. 
rías de información que no incluimos en el presente artículo, pero que son ya una realidad en los Accounting Dictionaries que ofrecen 23 opciones de búsqueda (Bergenholtz, 2012; Fuertes-Olivera y Tarp, 2014).

3.3.3. Elementos estructurales: en este apartado nos centramos en dos elementos de marcado carácter pedagógico que no forman parte de la microestructura de la obra sino que se presentan como herramientas conformadoras de la estructura global del diccionario electrónico que proponemos. En concreto se trata de la Introducción Sistemática y de la Guía de Uso.

- Introducción Sistemática: Fuertes-Olivera y Tarp (2011:1) la definen como "componentes externos adecuados para ayudar a los usuarios de los diccionarios especializados ya que funcionan como una especie de manual con el que un usuario no experto [...] puede adquirir un conocimiento adecuado de un dominio". Nuestra propuesta aboga por la inclusión de una Introducción Sistemática que tiene una doble función pedagógica: por un lado funciona como un libro de texto que el alumno puede consultar para solucionar necesidades de tipo cognitivo que surjan en la situación de recepción de texto y, por otro lado, es un elemento contextualizador del vocabulario especializado con la gran ventaja de que está diseñado con un nivel adecuado al usuario de nivel intermedio en cuanto a conocimiento de la lengua inglesa. Para alumnos con un menor nivel el diccionario proporciona explicaciones adjuntas a cada término en un lenguaje más básico y con la posibilidad de traducción al español; y para los de mayor nivel de conocimientos lingüísticos la forma ideal de proporcionar contextos semánticos es la inclusión de enlaces a páginas y materiales reales en Internet.

Proponemos que esta introducción tenga como idioma predeterminado el inglés y, al estar elaborada en un lenguaje de nivel intermedio y con un estilo pedagógico, que no disponga de traducción completa al español sino de una herramienta de traducción sencilla que traduzca un término si el cursor se detiene sobre él ${ }^{13}$.

- Guía de Uso: algunos estudios ${ }^{14}$ demuestran que hay usuarios que emplean menos tiempo en encontrar datos en obras impresas que en las electróni-

13. Para un ejemplo, ver la Wikipedia.

14. Bergenholtz, Henning (2011): "Access to and Presentation of Needs-Adapted Data in Monofunctional Internet Dictionaries" en Pedro A. Fuertes-Olivera y Henning Bergenholtz (eds.). ELexicography. The Internet, Digital Initiatives and Lexicography. Londres y Nueva York: Continuum, pp. 30-53. 
cas. Es lógico asumir que sin formación y práctica continua el usuario no lograría los mismos resultados con las nuevas herramientas electrónicas. Verlinde (2011:275) por su parte afirma que: "[ ... users do not always know how to use and interpret lexicographic resources that differ significantly from traditional paper dictionaries or well-known electronic dictionaries" y Lew y Galas (2008) afirman que aumentando las habilidades de los usuarios en el manejo de los diccionarios se reduciría la brecha entre la complejidad de la estructura de los diccionarios y la falta de habilidades en su manejo del usuario medio.

Por todo ello, creemos que una Guía de Uso accesible desde todas las pantallas del diccionario es imprescindible y también estamos a favor de una sesión práctica en la que el profesor indique al alumno usuario la forma adecuada de acceso a la obra y a los datos que necesita. Por otro lado, no todos los alumnos tienen la misma capacidad ni disposición para el aprendizaje de las nuevas tecnologías (ni de ninguna materia), por lo que una obra de carácter pedagógico debe intentar llegar a todos los usuarios potenciales, no solo a los mejor dotados.

\section{Ejemplo del resultado de búsqueda}

A continuación (v. figura 1) presentamos un ejemplo de los resultados de la búsqueda del término tariff. En primer lugar el alumno introduce el término tariff en la barra de búsqueda y selecciona la opción is. 


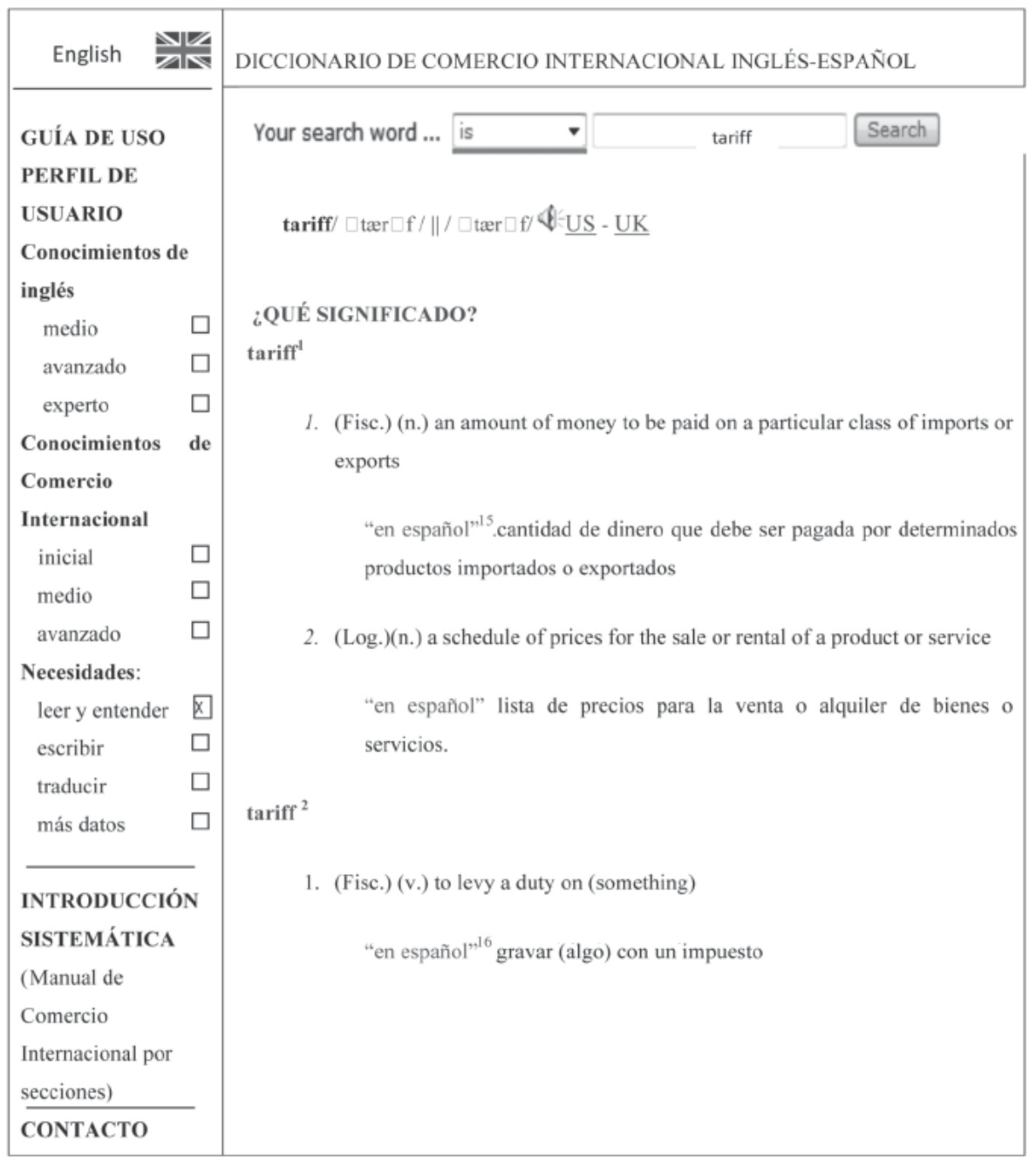

Figura 1. Resultado de la búsqueda de tariff; primera pantalla.

A continuación el usuario elige la primera acepción del primer homónimo obteniendo el resultado que aparece en la figura 2.

15. Recordamos que esta traducción del ejemplo aparece solo si se pincha el enlace "en español". 16. Recordamos que esta traducción del ejemplo aparece solo si se pincha el enlace "en español". 


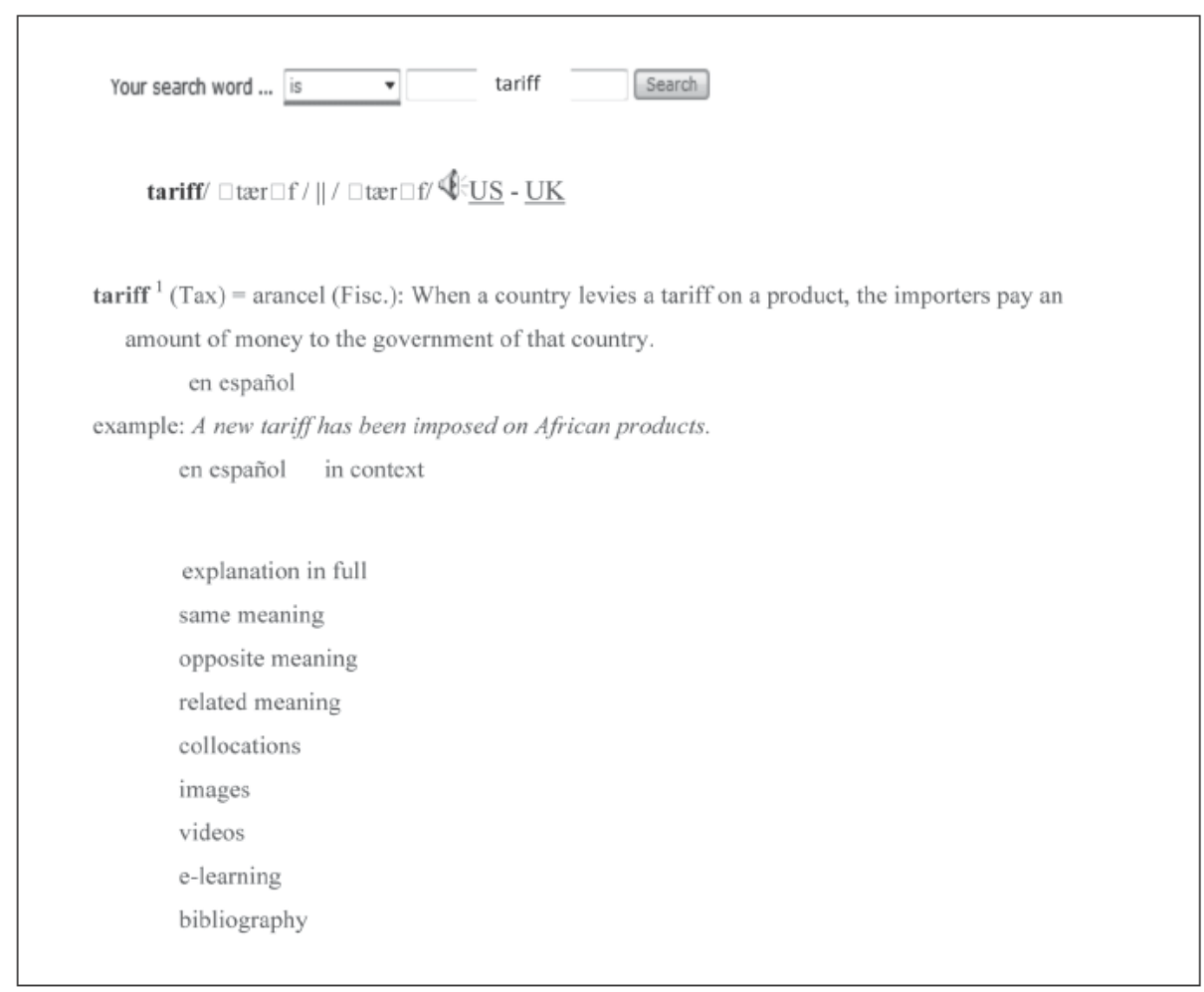

Figura 2. Resultado después de elegir la primera acepción del primer homónimo.

Por último, el usuario activa aquellos enlaces que necesite para solucionar sus necesidades lexicográficas. Ofrecemos en la figura 3 una vista de lo que aparecería en pantalla activando todos los enlaces (excepto las marcas de materia, que remitirían a la Introducción Sistemática y activarían mapas conceptuales). 


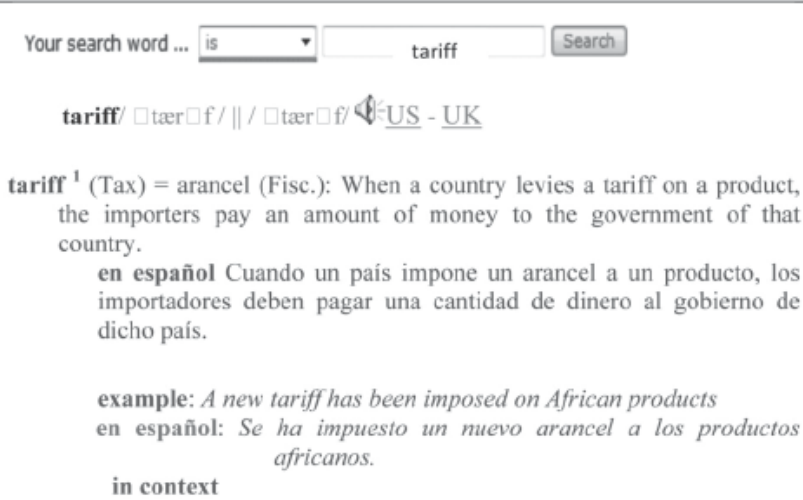

http://www.americaneconomicalert.org/news home.asp?NTID=90 http://www.bbc.co.uk/search/news/?q=tariff http://www.internationaltrade.co.uk/news.php?NID=65\&Title=Am erican + Cheese+Rules + Grate+on+French

explanation in full

History

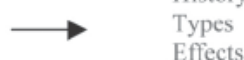

Effects

Legislation

Economic analysis

Political analysis

All

External links

same meaning customs tariff

opposite meaning (adj) non-tariff

related meaning tax. fee, cost, duty, excise, import, levy, price, rate, toll

collocations remove tariffs, tariff applied to, raise the tariff, a reduction in a tariff

images

http://www.treasury,govt.nz/publications/research-policy/wp/2007/07.

05/03.htm

http://www.google.es/search?hl=es\&rlz=1T4ADFA_esES366ES366\&q=tarif f\&um $=1$ \&ie $=$ UTF .

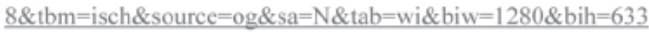
videos

http://www.google.es/search?hl=es\&rlz=1T4ADFA esES366

ES366\&q=tariff\&um $=1 \&$ biw $=1280 \&$ bih $=633 \& i e=U T F-$

$\underline{8 \& \text { tbo }=u \& \text { tbm }=\text { vid \& source }=0 \text { og } \& a=N \& \text { tab }=i v}$

\section{e-learning}

http://ec.europa.eu/taxation customs/common/elearning/general overview/in dex en.htm

http://www,wcoomd.org/learning customshome customselearninghome det ailshome.htm

bibliography

Figura 3. Resultado de la búsqueda al activar todos los enlaces. 


\section{Conclusiones}

La conclusión principal y más relevante que extraemos de nuestro estudio es la constatación de la idoneidad de la Teoría Funcional de la Lexicografía para aportar un enfoque pedagógico a las obras de referencia especializadas. Dentro de este marco teórico hemos podido definir con precisión la situación extralexicográfica concreta del alumno al que va dirigida nuestra propuesta de diccionario, hemos analizado el perfil de este usuario siguiendo las pautas expuestas por Sven Tarp en forma de preguntas claves y hemos podido matizar este perfil con preguntas adicionales que concretan las necesidades lexicográfica y pedagógicamente relevantes del alumno universitario español de Comercio Internacional.

Por otro lado, hemos explorado formas de presentar los datos en la interfaz de manera que se potencie el aprendizaje de contenidos especializados a la par que la adquisición de habilidades lingüísticas, teniendo como directriz de este proceso el criterio de relevancia aplicado en las obras que se desarrollan dentro del marco de la Teoría Funcional de la Lexicografía. En este sentido cabe destacar que si bien la propuesta que aquí presentamos no está todavía materializada en un producto lexicográfico tangible, sí existen obras lexicográficas elaboradas siguiendo los postulados de la Teoría Funcional de la Lexicografía que han demostrado en la práctica su idoneidad para satisfacer las necesidades lexicográficamente relevantes de los usuarios. Entre ellas cabe destacar los diccionarios de contabilidad o accounting dictionaries, un conjunto de 27 diccionarios de Internet que incorporan todas las novedades conocidas en este campo de investigación. Están diseñados y construidos por expertos del Centre for Lexicography de la Universidad de Aarhus, de la Universidad de Valladolid y de la empresa danesa Ordbogen.com.

Por último, mediante la Guía de Uso y la Introducción Sistemática que proponemos creemos que estamos aportando herramientas pedagógicas tanto para el alumno como para el profesor, los cuales necesitan nuevos materiales didácticos adaptados a los nuevos currículos, formas de enseñanza y objetivos marcados por los Acuerdos de Bolonia.

\section{Bibliografía}

ANDERSEN, B. y FUERTES-OLIVERA, P. A. (2009). "The Application of Function Theory to the Classification of Monolingual Business Dictionaries". Lexicographica 25: 213-239. 
ANDERSEN, B. y ALMIND, R. (2011). "The Technical Realization of Three Monofunctional Phrasal Verb Dictionaries" en E-Lexicography. The Internet, Digital Initiatives and Lexicography. (Ed. P. A. Fuertes-Olivera y H. Bergenholtz). Londres: Continuum.

Base de datos terminológica de la OMC. <http://wtoterm.wto.org/multiterm/ index.mto?locale=en>. (Acceso: marzo, 2012).

BERGENHOLTZ, H. (2011). "Access to and Presentation of Needs-Adapted Data in Monofunctional Internet Dictionaries" en E-Lexicography. The Internet, Digital Initiatives and Lexicography. (Ed. P. A. Fuertes-Olivera y H. Bergenholtz). Londres: Continuum.

BERGENHOLTZ, H. (2012). "Concepts for monofuctional accounting dictionaries". Terminology 18 (2): 243-263.

BERGENHOLTZ, H. y NIELSEN, S. (2006). "Subject-field components as integrated parts of LSP diccionaries". Terminology 12 (2): 281-303.

BERGENHOLTZ, H., NIELSEN, S. y TARP, S. (2009). Lexicography at a Crossroads. Dictionaries and Encyclopedias Today, Lexicographical Tools Tomorrow. Berna: Peter Lang.

BERGENHOLTZ, H. y TARP, S. (2003). "Two opposing theories: On H.E. Wiegand's recent discovery of lexicographic functions". Hermes. Journal of Linguistics 31: 171-196.

BERGENHOLTZ, H. y TARP, S. (2004). "The concept of dictionary usage". Nordic Journal of English Studies 3: 23-36.

BERGENHOLTZ, H. y TARP, S. (2005a). "Wörterbuchfunktionen” en Schreiben, Verstehen, Übersetzen, Lernen. Zu ein-und zweisprachigen Wörterbüchern mit Deutsch. (Ed. I. Barz, H. Bergenholtz y J. Korhonen). Frankfurt am Main: Peter Lang.

BERGENHOLTZ, H. y TARP, S. (2005b). "Electronic dictionaries: Old and new lexicographic solutions". Hermes. Journal of Linguistics 34: 7-9.

BOTHMA, T. J. D. (2011). "Filtering and Adapting Data and Information in an Online Environment in Response to User Needs" en E-Lexicography. The Internet, Digital Initiatives and Lexicography. (Ed. P. A. Fuertes-Olivera y H. Bergenholtz). Londres: Continuum.

FERNÁNDEZ, T. y FABER, P. (2011). "The Representation of Multidimensionality in a Bilingualized English-Spanish Thesaurus for Learners in Architecture and Building Construction". International Journal of Lexicography 24 (2): 198-225.

FL: WIKIMEDIA FOUNDATION (2004): Wikipedia: The free encyclopedia. $<$ http://www.wikipedia.org $>$. (Acceso: marzo, 2012). 
FL: WIKIMEDIA FOUNDATION (2004): Wiktionary: The free dictionary. $<\mathrm{http}$ ://www.wiktionary.org/>. (Acceso: marzo, 2012).

FUERTES-OLIVERA, P. A. (2010). "Lexicography for The Third Millennium: Free Institutional Internet Terminological Dictionaries for Learners" en Specialised Dictionaries for Learners. (Ed. P. A. Fuertes-Olivera). Berlin: De Gruyter.

FUERTES-OLIVERA, P. A. y ARRIBAS-BAÑO, A. (2008). Pedagogical Specialised Lexicography. The representation of meaning in English and Spanish business dictionaries. Amsterdam/Philadelphia: John Benjamins. (Terminology and Lexicography in Research and Practice, 11).

FUERTES-OLIVERA, P. A. y BERGENHOLTZ, H. (ed.) (2011). E-Lexicography. The Internet, Digital Initiatives and Lexicography. Londres: Continuum.

FUERTES-OLIVERA, P. A. y TARP, S. (2011). "Lexicography for The Third Millennium: Cognitive-oriented Specialised Dictionaries for Learners". Iberica. Journal of the European Association of Languages for Specific Purposes 21.

FUERTES-OLIVERA, P. A. y TARP, S. (2014). Theory and Practice of Specialised Online Dictionaries: Lexicography versus Terminography. Berlin: De Gruyter.

FUNDACIÓN BBVA (2010). Estudio internacional Fundación BBVA sobre estudiantes universitarios de seis paises europeos. $<\mathrm{http}$ ://www.fbbva.es/TLFU/ dat/resultados_universitarios_2010.pdf > . (Acceso: 3 noviembre 2011).

GOUWS, R. H. (2011). "Learning, Unlearning and Innovation in the Planning of Electronic Dictionaries" en E-Lexicography. The Internet, Digital Initiatives and Lexicography. (Ed. P. A. Fuertes-Olivera y H. Bergenholtz). Londres: Continuum.

HALBACH, A., LÁZARO, A. y PÉREZ GUERRA, J. (2010). "La acreditación del nivel de lengua inglesa en las universidades españolas". <http://www. revistaeducacion.mec.es/doi/362_154.pdf > . (Acceso: 8 noviembre 2012) y $<$ http://www.britishcouncil.org/spain_informe_acreditacion_ingles_ universidades_espanolas.pdf $>$. (Acceso: 8 noviembre 2011).

HEID, U. (2011). "Electronic Dictionaries as Tools: Toward An Assesment of Usability" en E-Lexicography. The Internet, Digital Initiatives and Lexicography. (Ed. P. A. Fuertes-Olivera y H. Bergenholtz). Londres: Continuum.

IATE: Base de datos terminológica para la Unión Europea. <http://iate. europa.eu/iatediff/SearchByQuery.do>. (Acceso: marzo, 2012).

LANDAU, S. I. (2001). Dictionaries: The Art and Craft of Lexicography. Cambridge: Cambridge University Press. 
LEW, R. (2011). “Online Dictionaries of English” en E-Lexicography. The Internet, Digital Initiatives and Lexicography. (Ed. P. A. Fuertes-Olivera y H. Bergenholtz). Londres: Continuum.

LEW, R. y GALAS, K. (2008). Can Dictionary Skills Be Taught? The Effectiveness of Lexicographic Training for Primary-School-Level Polish Learners of English. <http://www.staff.amu.edu.pl/ rlew/pub/>. (Acceso: 3 noviembre 2011).

NIELSEN, S. y ALMIND, R. (2011). "From data to dictionary" en E-Lexicography. The Internet, Digital Initiatives and Lexicography. (Ed. P. A. Fuertes-Olivera y H. Bergenholtz). Londres: Continuum.

NIELSEN, S., MOURIER, L. y BERGENHOLTZ, H. (2003-2007). Accounting Dictionaries: Danish Accounting Dictionary; English-Accounting Dictionary; Danish-English Accounting Dictionary; English-Danish Accounting Dictionar. $<\mathrm{http}: / /$ www.regnskabsordbogen.dk/>. (Acceso: marzo, 2012).

NIELSEN, S., MOURIER, L., BERGENHOLTZ, H., FUERTES OLIVERA, P., GORDO GÓMEZ, P., NIÑO AMO, M., RIOS RODICIO, A. DE LOS, SASTRE RUANO, A., TARP, S.,VELASCO SACRISTÁN, M. (2009). El Diccionario Inglés-Español de Contabilidad. <http://www.lemma.com>. (Acceso: julio, 2013).

NIELSEN, S. y TARP, S. (eds.) (2009). Lexicography in the $21^{\text {st }}$ Century. In Honour of Henning Bergenholtz. Amsterdam: John Benjamins. (Terminology and Lexicography in Research and Practice, 12).

TARP, S. (2008). Lexicography in the Borderland between Knowledge and NonKnowledge. General Lexicographical Theory with Particular Focus on Learner's Lexicography. Tübingen: Niemeyer.

TARP, S. (2010). "Functions of Specialised Learners' Dictionaries" en Specialised Dictionaries for Learners. (Ed. Pedro A. Fuertes-Olivera). Berlin: De Gruyter.

VERLINDE, S. (2011). "Modelling Interactive Reading, Translation and Writing Assistants" en E-Lexicography. The Internet, Digital Initiatives and Lexicography. (Ed. P. A. Fuertes-Olivera y H. Bergenholtz). Londres: Continuum.

Wordreference.com: diccionarios de español, ingles, francés y portugués. $<\mathrm{http}: / / w w w . w o r d r e f e r e n c e . c o m />$. (Acceso: marzo, 2012).

ZGUSTA, L. (1971). Manual of lexicography. The Hague: Mouton. (Janua Linguarum. Series maior, 39). 